\title{
nombalina
}

(8)

\section{Marziale catulliano}
Autor(es):
Fedeli, Paolo
Instituto de Estudos Clássicos, Centro de Estudos Clássicos e
Publicado por: Humanísticos; Departamento de Estudos Clássicos, Centro de Estudos Clássicos

\author{
URL \\ persistente: \\ URI:http://hdl.handle.net/10316.2/34635 \\ DOI: \\ DOI:http://dx.doi.org/10.14195/978-989-26-0901-0_9
}

Accessed : $\quad$ 26-Apr-2023 11:22:24

A navegação consulta e descarregamento dos títulos inseridos nas Bibliotecas Digitais UC Digitalis, UC Pombalina e UC Impactum, pressupõem a aceitação plena e sem reservas dos Termos e Condições de Uso destas Bibliotecas Digitais, disponíveis em https://digitalis.uc.pt/pt-pt/termos.

Conforme exposto nos referidos Termos e Condições de Uso, o descarregamento de títulos de acesso restrito requer uma licença válida de autorização devendo o utilizador aceder ao(s) documento(s) a partir de um endereço de IP da instituição detentora da supramencionada licença.

Ao utilizador é apenas permitido o descarregamento para uso pessoal, pelo que o emprego do(s) título(s) descarregado(s) para outro fim, designadamente comercial, carece de autorização do respetivo autor ou editor da obra.

Na medida em que todas as obras da UC Digitalis se encontram protegidas pelo Código do Direito de Autor e Direitos Conexos e demais legislação aplicável, toda a cópia, parcial ou total, deste documento, nos casos em que é legalmente admitida, deverá conter ou fazer-se acompanhar por este aviso.

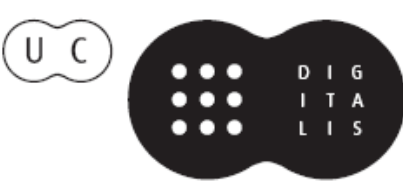




\section{Toto notus in orbe Martialis}

\section{Celebração de Marcial \\ 1900 anos após a sua morte}

Coordenação

Cristina de Sousa Pimentel

Delfim F. Leão

José Luís L. Brandão

Instituto de Estudos Clássicos

Centro de Estudos Clássicos e Humanísticos

UNIVERSIDADE DE COIMBRA
Departamentō de Estudos Clássicos Centro de Estudos Clássicos UNIVERSIDADE DE LISBOA 


\section{MARZIALE CATULLIANO}

PAOLO FEDELI

Università di Bari

Abstract: This contribution tries to analyze the influence of Catullus' poetry on Martial's epigrammatic production.

1. È noto che per l'epigramma di Marziale la poesia di Catullo costituisce un punto di riferimento costante: da lui viene assimilata la predilezione per lo svolgimento vivace e per i toni polemici, che tuttavia non sconfinano mai nella violenta aggressione personale. Da Catullo egli eredita anche la varietà metrica: non a caso, infatti, i metri preferiti oltre al distico elegiaco (gli endecasillabi faleci e gli scazonti) rinviano alle scelte metriche predilette da Catullo nelle nugae. Tutto ciò è dato per scontato, ma in mancanza di una seria indagine complessiva, ${ }_{r}^{1}$ qualsiasi discorso in merito ai debiti reali del poeta epigrammatico di età imperiale nei confronti del suo predecessore di età repubblicana rischia di rimanere parziale e provvisorio. Così sarà anche per la mia indagine, che vuole fare il punto della situazione e introdurre un po' di chiarezza nella sempre più sfrenata ricerca di paralleli che molto spesso sono in realtà pseudoparalleli.

L'adesione di Marziale a Catullo è proclamata in modo chiaro sin dall'epistola proemiale del I libro: lascivam verborum veritatem, id est epigrammaton linguam, excusarem, si meum esset exemplum: sic scribit Catullus, sic Marsus, sic Pedo, sic Gaetulicus, sic quicumque perlegitur: benché qui Catullo apra una lista di poeti, che include anche Domizio Marso,

${ }^{1}$ Parziali e provvisorie sono, dopo Paukstadt (1876), le ricerche di Ferguson (1963) 3-15, di Offermann (1980) 107-139, di Newman (1990) 75-103, di Swann (1998) 48-58. 
Albinovano Pedone e Cornelio Lentulo Getulico, è lui che Marziale considera il modello della propria poesia epigrammatica. Le numerose attestazioni parlano chiaro, e basterà limitarsi alle più significative: in 4.14.13 Catullo è definito tener, mentre è detto tenuis in 10.103.5 (con un preciso riferimento di poetica alla tenuitas della sua poesia) e doctus in 7.99 .9 (con un'allusione alla necessaria dote della doctrina, secondo la poetica alessandrino-neoterica), dove Marziale si augura di non sfigurare nei confronti dei suoi carmi; in 5.5.5-6, poi, rivolgendosi a Sesto, direttore della biblioteca di Minerva sul Palatino, lo prega di trovare uno spazio per le sue poesie accanto a quelle di Albinovano Pedone, di Domizio Marso e, naturalmente, di Catullo. Nell'epigramma 10.78, infine, nel rivolgersi a Macro si augura di essere letto fra i poeti antichi e da lui posposto al solo Catullo (vv. 14-16):

sic inter veteres legar poetas

nec multos mihi praeferas priores,

uno sed tibi sim minor Catullo.

È significativo che Marziale, in apertura del suo primo libro di epigrammi, abbia sentito l'esigenza di giustificare il contenuto non propriamente casto dei suoi versi, e lo abbia fatto con un esplicito richiamo ad un'analoga presa di posizione di Catullo nei confronti dei benpensanti, che certamente si sarebbero scandalizzati di fronte ai suoi versi. Nel c. 16 Catullo, dopo un colorito esordio con la minaccia di punizioni sessuali nei confronti dei calunniatori Furio e Aurelio, aveva contrapposto i propri versi lascivi alla sua vita, che invece era casta:

Pedicabo ego vos et irrumabo,

Aureli pathice et cinaede Furi,

qui me ex versiculis meis putastis,

quod sunt molliculi, parum pudicum.

Nam castum esse decet pium poetam

ipsum, versiculos nihil necesse est;

qui tum denique habent salem ac leporem,

si sunt molliculi ac parum pudici,

et quod pruriat incitare possunt,

non dico pueris, sed his pilosis

qui duros nequeunt movere lumbos.

Vos, quod milia multa basiorum

legistis, male me marem putatis?

Pedicabo ego vos et irrumabo. 
Accusato, dunque, dai due detrattori di impudicizia a causa del tono dei suoi versi (vv. 3-4), Catullo si difende sostenendo che il poeta deve essere, sì, casto, ma nel suo comportamento di vita, non necessariamente nei suoi versi. In tal modo egli contrappone i suoi versi lascivi alla figura convenzionale del pius poeta (v. 5). Marziale riprende lo stesso concetto e, basandosi su una scoperta allusività nei confronti del contesto catulliano, lo mette in chiaro sin dall'inizio della sua raccolta epigrammatica: rivolgendosi all'imperatore Domiziano, che immagina quale lettore dei suoi versi, lo esorta a non considerarli con occhio malevolo a causa del loro carattere lascivo (1.4):

Contigeris nostros, Caesar, si forte libellos, terrarum dominum pone supercilium.

Consuevere iocos vestri quoque ferre triumphi materiam dictis nec pudet esse ducem.

Qua Thymelen spectas derisoremque Latinum, 5 illa fronte precor carmina nostra legas.

Innocuos censura potest permittere lusus: lasciva est nobis pagina, vita proba.

Qui è il verso conclusivo a fornire l'identica giustificazione di cui si era servito Catullo, grazie alla contrapposizione fra la pagina e la vita. Non contento, però, di una tale presa di posizione, Marziale decide di ribadirla, nel corso dello stesso I libro, rivolgendosi a un non meglio identificabile Cornelio (1.35):

Versus scribere me parum severos nec quos praelegat in schola magister,

Corneli, quereris: sed hi libelli, tamquam coniugibus suis mariti, non possunt sine mentula placere.

Quid si me iubeas talassionem verbis dicere non talassionis?

Quis Floralia vestit et stolatum. permittit meretricibus pudorem? Lex haec carminibus data est iocosis, ne possint, nisi pruriant, iuvare.

Quare deposita severitate parcas lusibus et iocis rogamus, nec castrare velis meos libellos.

Gallo turpius est nihil Priapo. 
A Cornelio, che l'accusa di scrivere versi poco castigati e impresentabili a scuola, Marziale risponde che senza un contenuto lascivo essi non avrebbero alcun successo: e s'inventa una lex carminis, secondo la quale i versi giocosi non possono piacere se non sono lascivi (vv. 10-11). Cornelio, quindi, messo da parte il suo piglio severo dovrà guardarsi bene dal castrare i libretti del poeta epigrammatico, perché non c'è nulla di più immondo di un Priapo castrato (vv. 12-15). ${ }^{2}$ In questo caso, al di là del contenuto, la spia dell'allusione al contesto catulliano è nel v. 5 ( $h i$ libelli) non possunt sine mentula placere e nel v. 11 ne possint, nisi pruriant, iuvare, che rinviano ai vv. 7-9 del c. 16 di Catullo: qui tum denique habent salem ac leporem, I si sunt molliculi ac parum pudici, I et quod pruriat incitare possunt. ${ }^{3}$

2. Ma vediamo più da vicino i punti di contatto più evidenti fra la poesia di Marziale e quella di Catullo. Nella tecnica del verso Catullo è stato senza alcun dubbio il modello di Marziale, in particolare per quanto riguarda la struttura dell'endecasillabo falecio. ${ }^{4}$ Comune a entrambi è la tecnica di collocare due termini corrispondenti l'uno all'inizio, l'altro alla fine del verso, con una particolare preferenza per la collocazione anticipata dell'aggettivo (cf. e.g. Catullo $1.4 \mathrm{me}$ a s esse aliquid putare $n u g a s \sim$ Marziale 1.1.3 argutis epigrammaton libellis). ${ }^{5}$ Forti analogie sono anche nella predilezione per la collocazione chiastica dei termini nell'endecasillabo falecio (cf. e.g. Catullo 7.6 et Batti veteris sacrum sepulcrum Marziale 1.117.6 rasum pumice purpuraque cultum $)^{6}$ o nella ripetizione di parole nella stessa posizione metrica (cf. e.g. Catullo 3.3-4 passer mortuus est meae puellae, / passer, deliciae meae puellae Marziale 1.7.1-5 Stellae delicium mei colu $\mathrm{m} b$ a / ...quanto passere maior est c o lu $m b a)^{7}$ o nel poliptoto in due endecasillabi successivi (cf. e.g. Catullo 23.5-6 est pulcre tibi cum tuo paren te / et cum coniuge lignea

2 In merito cf. Hallett (1996) 321-344.

${ }^{3}$ Sul parallelo fra Marziale 1.35 e il c. 16 di Catullo, oltre al commento di Citroni (1975) 32-33 cf. Offermann (1980) 114.

${ }^{4}$ Ovviamente, qui e in seguito, mi limiterò solo ad alcuni esempi illustrativi.

${ }^{5}$ Cf. Paukstadt (1876) 30, Citroni (1975) 15.

${ }^{6}$ Altri esempi in Marziale sono citati da Citroni (1975) 358 e da Salemme (1976) 49.

${ }^{7}$ Cf. Paukstadt (1876) 31, Citroni (1975) 42. 
parentis Marziale 1.73-4 vicit, Maxime, passerem C a t ulli.l Tanto Stella meus tuo $\mathrm{Cat} u(l o)^{8} \mathrm{o}$, infine, nella consuetudine di aprire e chiudere un carme con l'identico verso (cf. e.g. Catullo 52.1-4 quid est, Catulle, quid moraris emori?/ ...Quid est, Catulle, quid moraris emori? Marziale 2.6.1-17 i, nunc, edere me iube libellos). ${ }^{9} \mathrm{Se}$, poi, dall'endecasillabo falecio si passa al giambo zoppo o scazonte, è notevole che, come già Catullo nel c. 8 (miser Catulle, desinas ineptire) e nel c. 31 (Paene insularum, Sirmio, insularumque), Marziale non se ne serva esclusivamente in carmi d'invettiva o comunque aggressivi (cf. infatti, e.g. 3.58 Baiana nostri villa, Basse, Faustini). ${ }^{10}$

E ancora: se Catullo nel c. 78 fa iniziare allo stesso modo i tre distici di cui è costituito l'epigramma (v. 1 Gallus habet fratres, v. 3 Gallus homo est bellus, v. 5 Gallus homo est stultus) o nel c. 103 fa terminare nell'identico modo i due distici (vv. 2 e 4 saevus et indomitus), Marziale riprende ed esaspera questa tecnica sia in 1.77, dove tutti gli scazonti terminano con Charinus et tamen pallet (vv. 1-5), con una ironica variazione nell'ultimo verso (v. 6 Charinus lingit et tamen pallet), sia in 11.57.3-11, in cui è nec ad aprire tutti i versi dell'epigramma. Se, infine, Catullo predilige per la sua particolare musicalità la collocazione dei comparativi in clausola nell'endecasillabo falecio (mi limito a citare i casi di 3.2 et quantum est hominum venustiorum e di 5.2 rumoresque senum severiorum), non sorprende che identica sia la scelta di Marziale (anche per lui mi limito a citare due casi: 1.115.4 sed quandam volo nocte nigriorem, 5.2.3 tu quem nequitiae procaciores)..$^{11}$

Se dalla struttura si passa al lessico, è facile individuare una serie di termini che Marziale mostra di riprendere direttamente da Catullo. È questo il caso, ad esempio, di anus, che Marziale adopera nel rarissimo valore di aggettivo in 1.39 .2 quales prisca fides famaque novit anus; lo stesso aveva fatto Catullo sia in 78.4 fama loquatur anus sia in 68.46 carta loquatur anus, un emistichio che Marziale riprende con una lieve variazione in 12.3 (4).4 cartaque dicet anus. ${ }^{12}$ L'aggettivo bellus, chiaro sinonimo colloquiale di pulcher, che compare spesso sia in Catullo sia in Marziale, «è uno dei casi

\footnotetext{
${ }^{8}$ Cf. Citroni (1975) 135.

${ }^{9}$ Cf. Paukstadt (1876) 34, Salemme (1976) 51.

${ }^{10}$ Numerosi altri esempi in Marziale sono citati da Salemme (1976) 54.

${ }^{11}$ Altri casi sono citati dalla Bonvicini (1986) 31-35.

${ }^{12}$ In proposito cf. Citroni (1975) 125-6, a 1.39.2.
} 
più significativi di termini di coloritura sicuramente quotidiana che Marziale usi con particolare frequenza»,$^{13}$ Marziale avrà subito, con ogni probabilità, l'influsso dell'uso molto ampio che ne fa Catullo. Il sostantivo astratto esuritio, che compare due volte in Marziale (1.99.10; 5.78.18), è termine molto raro: tuttavia esso compare due volte anche in Catullo $(21.1 ; 23.14)$, ed in entrambi i casi alla fine del falecio, proprio come in Marziale. Per quanto riguarda, poi, un termine osceno come fututio, Marziale (1.106.6 certae nequitias fututionis) lo riprende da un contesto catulliano di contenuto analogo (32.8 novem continuas fututiones). Ma dal suo modello Marziale trae anche termini solenni, come il composto veridicus (5.1.3 seu tua veridicae discunt responsa sorores), che Catullo aveva già usato per le profezie delle Parche nel c. 64 (v. 306 veridicos Parcae coeperunt edere cantus, 325-6 accipe, quod laeta tibi pandunt luce sorores, / veridicum oraclum). Ma anche l'unica attestazione del raro e arcaico pote in Marziale (9.15.2 quid pote simplicius?) mostra il chiaro influsso di Catullo, che per parte sua lo predilige (5 attestazioni), mentre si tratta di forma praticamente ignorata dai poeti del I sec. a.C. ${ }^{14}$ Da ultimo ho lasciato il termine nugae, che in Catullo definisce la poesia leggera sin dal carme di dedica a Cornelio Nepote (1.3-4 namque tu solebas I meas esse aliquid putare nugas): non solo Marziale chiama spessissimo nello stesso modo le sue poesie (19 occorrenze), ma di solito quando usa il termine lo fa con esplicito riferimento al lessico catulliano. ${ }^{15}$

3.1. Certamente più rilevanti sono $\mathrm{i}$ casi in cui uno o più epigrammi di Catullo servono da modelli a uno o a più epigrammi di Marziale. Un esempio significativo è costituito dall'accurata descrizione dell'aspetto esteriore del liber, che Catullo dà nel carme di dedica a Cornelio Nepote (c. 1):

Cui dono lepidum novum libellum

arida modo pumice expolitum?

Corneli, tibi: namque tu solebas

meas esse aliquid putare nugas

iam tum, cum ausus es unus Italorum

${ }^{13}$ Citroni (1975) 47-48.

${ }^{14}$ Cf. Henriksén (1998-9) I. 107.

${ }^{15}$ Cf. Citroni (1975) 346, a 1.113 .6 e Grewing (1998) 55. 
omne aevum tribus explicare cartis doctis, Iuppiter, et laboriosis.

Quare habe tibi quidquid hoc libelli qualecumque; quod, o patrona virgo, plus uno maneat perenne saeclo.

L'aspetto esteriore di un liber, quello di Suffeno, un poetastro che scrive migliaia di versi, è descritto da Catullo anche nei vv. 1-8 del c. 22:

cartae regiae, novi libri,

novi umbilici, lora rubra membranae,

derecta plumbo et pumice omnia aequata.

Fra le varie descrizioni che dei suoi libelli ci dà Marziale, oltre a quella di 5.6.13-14 (numquam grandia nec molesta poscit / quae cedro decorata purpuraque / nigris pagina crevit umbilicis) la più notevole è fornita dall'epigramma 3.2:16

Cuius vis fieri, libelle, munus?

Festina tibi vindicem parare, ne nigram cito raptus in culinam cordylas madida tegas papyro vel turis piperisve sis cucullus.

Faustini fugis in sinum? Sapisti.

Cedro nunc licet ambules perunctus et frontis gemino decens honore pictis luxurieris umbilicis, et te purpura delicata velet, 10 et cocco rubeat superbus index. Illo vindice nec Probum timeto.

È evidente che l'esordio dell'epigramma di Marziale riprende le stesse movenze del c. 1 di Catullo, con una proposizione interrogativa in cui il poeta neoterico si chiede a chi dedicare il suo libro di poesie (cui dono lepidum novum libellum?), mentre Marziale preferisce rivolgere la domanda al libro stesso (cuius vis fieri, libelle, munus?). Il destinatario del libro, poi, viene posto da Catullo all'inizio del v. 3 (Corneli, tibi), da Marziale all'inizio del v. 6 (Faustini fugis in sinum?): apparentemente la funzione di Corneli e di Faustini sembra la stessa, ma in realtà il primo è

${ }^{16}$ Sull'epigramma di Marziale cf. Offermann (1980) 107-9. 
un vocativo, il secondo un genitivo. Nei vv. 3-5, poi, nel far paventare al libro il rischio di divenire vile foglio per avvolgere tonnetti o per fare cartocci d'incenso e di pepe, Marziale abbandona il c. 1, ma non Catullo, perché la sua grottesca immagine ricorda quella che il poeta neoterico riserva nel c. 95 ai fogli di papiro contenenti gli Annali di Volusio, destinati ad essere usati per avvolgere gli sgombri (95.7-8 at Volusi Annales .../ ... laxas scombris saepe dabunt tunicas). Ancor più complessa è la conclusione dell'epigramma di Marziale, in cui i vv. 7-11, con la precisa descrizione dell'aspetto esteriore del libro, da un lato sembrano rinviare a Catullo 1.2, dall'altro, però, ricordano più da vicino i vv. 6-8 del c. 22. Ma non è finita qui, perché il v. 2 del carme catulliano (arida modo pumice expolitum) ritorna in Marziale 8.72.1-3 (nondum murice cultus asperoque / morsu pumicis aridi politus / Arcanum properas sequi, libelle), mentre i vv. 1-4 di Catullo trovano una più complessa ripresa nell'analoga descrizione dell'aspetto del libro in Marziale 4.10.1-4 (dum novus est nec adhuc rasa mihi fronte libellus, I pagina dum tangi non bene sicca timet, / $i$, puer, et caro perfer leve munus amico I qui meruit $n$ u g a s primus habere $m$ e a s) e il v. 8 (quare habe tibi quidquid hoc libelli qualecumque) nel v. 1 di Marziale 3.1, che pure è di tutt'altro contenuto e al carme di Catullo è accomunato solo dalla funzione incipitaria (hoc tibi quidquid id est longinquis mittit ab oris). ${ }^{17}$

3.2. I cc. 2-3 di Catullo, per il passero di Lesbia, sono stati ripresi in almeno 6 epigrammi di Marziale (1.7; 1.109; 4.14; 7.14; 11.6; 14.77). Un esempio significativo dell'adesione di Marziale alla poesia di Catullo è costituito dall'epigramma in endecasillabi faleci che celebra un carme di Arrunzio Stella (lo stesso personaggio le cui nozze con Violentilla sono celebrate da Stazio in Silvae 1.2, che è un epitalamio), a esaltazione della colomba tanto cara alla donna amata: a sua volta lo stesso Arrunzio Stella aveva preso a modello il carme di Catullo (1.7):

Stellae delicium mei columba,

Verona licet audiente dicam,

vicit, Maxime, passerem Catulli.

Tanto Stella meus tuo Catullo

quanto passere maior est columba.

${ }^{17}$ Cf. Paukstadt (1876) 10. 
Il carme di Marziale ha l'aspetto di un bigliettino poetico, che però non è inviato al destinatario dell'elogio (Arrunzio Stella), ma a un altro esponente del circolo letterario a cui entrambi afferivano (un Massimo di non certa identificazione). L'inizio costituisce un chiaro rinvio al primo dei due carmi catulliani per il passer di Lesbia, in quanto il v. 1 riprende il passer deliciae meae puellae di Catullo 2.1, con la raffinata sostituzione del plurale deliciae con il raro singolare delicium. Consapevole di dover affermare un concetto importante, Marziale introduce un'espressione parentetica (v. 2), che in realtà accresce il peso di ciò che sta per dire chiamando in causa addirittura Verona, la patria di Catullo. Dopoché il v. 3 ha formulato, con un tono che non ammette repliche, il giudizio di superiorità di Arrunzio Stella su Catullo, la particolarità dei due versi conclusivi risiede nel fatto che anch'essi sono costruiti su materiale catulliano, diverso però da quello del carme 2: Marziale combina qui la stessa clausola di endecasillabo di Catullo 38.1 (tuo Catullo) con un'allusione alla chiusa del noto e ambiguamente ironico epigramma catulliano di ringraziamento a Cicerone (il carme 49):

Disertissime Romuli nepotum, quot sunt quotque fuere, Marce Tulli, quotque post aliis erunt in annis, gratias tibi maximas Catullus agit, pessimus omnium poeta, 5 tanto pessimus omnium poeta, quanto tu optimus omnium patronus.

Apparentemente quello di Catullo è un biglietto di ringraziamento a Cicerone; ma che esso nasconda una forte carica ironica diviene manifesto se si considera che Catullo si appropria di una serie di espressioni di carattere ambiguo rivolgendole e applicandole al destinatario del carme (disertus, che in Cicerone indica il chiacchierone più che l'eloquens; Romuli nepotum che è designazione a tal punto altisonante per Romanus da far pensare a una presa in giro; optimus omnium patronus che può indicare sia il migliore di tutti gli avvocati sia il migliore avvocato di tutti gli accusati, e dunque non solo degli onesti ma anche dei disonesti). L'analogia fra la chiusa del carme di Catullo e quella dell'epigramma di Marziale è evidente, così come è notevole l'elegante disposizione chiastica nella chiusa dei versi dell'epigramma di Marziale (columba... Catulli... Catullo... columba, con l'ovvia esclusione dallo 
schema del v. 2 per la sua natura di inciso). Apparentemente il carme di Marziale si chiude con l'elogio di Arrunzio Stella, che egli considera molto più grande di lui. Tuttavia il richiamo allusivo invita i lettori a leggere il contesto dell'imitatore (Marziale) alla luce di quello del modello (Catullo). Se, dunque, s'interpreta la chiusa dell'epigramma di Marziale alla luce di quello di Catullo per Cicerone, l'ironia del modello si trasmette al contesto dell'imitatore: non solo il destinatario dell'epigramma, ma anche i lettori avrebbero capito che Marziale stava scherzosamente esagerando nella sua enfatizzazione della grandezza di Arrunzio Stella, che addirittura - a suo dire - vincerebbe il confronto con i più famosi versi di Catullo.

3.3. Concepito come un carme di dedica di un battello ormai a riposo, il c. 4 di Catullo descrive agli hospites (termine corrispondente al greco క́́vor degli epigrammi dedicatori) il lungo percorso che il phaselus ha compiuto dal suo luogo d'origine sino a quello in cui ora si gode un meritato riposo:

Phaselus ille, quem videtis, hospites,

ait fuisse navium celerrimus,

neque ullius natantis impetum trabis

nequisse praeterire, sive palmulis

opus foret volare sive linteo.

Et hoc negat minacis Hadiatrici

negare litus insulasve Cycladas

Rhodumque nobilem horridamque Thraciam

Propontida trucemve Ponticum sinum,

ubi iste post phaselus antea fuit

comata silva; nam Cytorio in iugo

loquente coma saepe sibilum edidit coma.

Amastri Pontica et Cytore buxifer,

ibi haec fuisse et esse cognitissima

ait phaselus: ultima ex origine

tuo stetisse dicit in cacumine,

tuo imbuisse palmulas in aequore,

et inde tot per impotentia freta

erum tulisse, laeva sive dextera

vocaret aura, sive utrumque Iuppiter

simul secundus incidisset in pedem;

neque ulla vota litoralibus deis 
sibi esse facta, cum veniret a mari novissimo hunc ad usque limpidum lacum.

Sed haec prius fuere: nunc recondita

senet quiete seque dedicat tibi,

gemelle Castor et gemelle Castoris.

Già Properzio (3.21.11-22) e Ovidio (Tristia 1.10) si erano ricollegati al carme catulliano: ${ }^{18}$ in entrambi i casi si tratta di un battello, quello che dovrebbe trasportare il poeta in un progettato viaggio in Grecia nel caso di Properzio, quello che conduce attraverso mari infidi verso la terra d'esilio nel caso di Ovidio. Anche Marziale si cimenta in una ripresa del c. 4, ma lo fa nell'epigramma 7.19 in un modo particolarmente complesso, su cui vale la pena di riflettere: ${ }^{19}$

Fragmentum quod vile putas et inutile lignum, haec fuit ignoti prima carina maris.

Quam nec Cyaneae quondam potuere ruinae frangere nec Scythici tristior ira freti, saecula vicerunt: sed quamvis cesserit annis, sanctior est salva parva tabella rate.

Anche l'epigramma di Marziale s'inserisce nella consuetudine dei carmi di dedica, come si deduce da sanctior, riferito a parva tabella, che fa pensare a una consacrazione di essa. In Marziale, però, non si è in presenza della dedica di un battello, bensì di un fragmentum (v. 1): si tratta, però, di un frammento a dir poco singolare, perché esso appartiene addirittura alla leggendaria nave Argo, quella di Giasone e degli Argonauti, che per prima osò solcare i mari (v. 2); di essa, appunto, Marziale ricostruisce l'itinerario attraverso le insidie delle acque e delle tempeste, che però non riuscirono a vincerla (vv. 3-4). Le vicissitudini del phaselus, che occupano un'ampia sezione del carme di Catullo (vv. 6-24), sono dunque riprese e condensate da Marziale in due soli versi. Ma le riprese vanno al di là della parte in cui è descritto il viaggio: esse sono

18 Sulle loro riprese del c. 4 si sofferma la Bonvicini (1995) 5-13.

19 Per un commento all'epigramma cf. Galán Vioque (2002) 153; utili osservazioni sono anche in Offermann (1980) 131-3. 
evidenti già nel verso di esordio, perché al catulliano phaselus ille, quem videtis Marziale allude sia con l'iniziale presentazione dell'oggetto che viene dedicato sia pure, con una analoga concentrazione stilistica, col deittico haec (v. 2) con cui egli presenta e indica visivamente ciò che è rimasto della prima carina; per di più la descrizione della sua straordinaria qualità (v. 2 haec fuit ignoti prima carina maris) corrisponde alla dote del phaselus che Catullo indica nel v. 2 del suo carme (ait fuisse navium celerrimus) e chiarisce, poi, nei vv. 3-4. Quando, poi, Marziale - conclusa rapidamente la narrazione delle peregrinazioni della nave Argo - osserva che saecula vicerunt e subito dopo riprende il motivo della vittoria del tempo in $s$ ed quamvis cesserit annis (v. 5), è chiaro che egli si ricollega al catulliano sed haec prius fuere: nunc recondita / senet quiete $(4,25-26) \mathrm{e}$ invita a interpretare il discusso verso conclusivo come dedica della parva tabella: proprio perché in Catullo il carme si conclude con la dedica del phaselus (v. 26 seque dedicat).

Tuttavia l'epigramma di Marziale è molto più complesso, tanto che a spiegarlo non basta solo il c. 4 di Catullo: non bisogna dimenticare, infatti, né la natura particolare dell'oggetto descritto (addirittura la nave Argo!), né la riduzione del suo viaggio lungo e pericoloso nel breve spazio di due versi. Credo, di conseguenza, che siano nel giusto quanti ritengono ${ }^{20}$ che l'epigramma di Marziale debba essere inteso anche alla luce della poetica callimachea della brevitas applicata ad argomenti epici: Marziale, cioè, vuole dimostrare che persino argomenti epici ben noti possono essere celebrati in pochi versi. La riduzione stessa dei 27 versi del c. 4 di Catullo a 6 versi è un esercizio di brevitas: ma nell'ambito di essa la condensazione delle peripezie della nave Argo assume un significato particolare, perché la scelta di un carme di Catullo appare funzionale a dimostrare come si possano cantare grandi argomenti epici in pochi versi. Catullo stesso, d'altronde, lo aveva già fatto nel c. 64 , che è un tipico esempio di epillio di fattura alessandrina. Per parte sua, Marziale vuole dimostrare di essere ancor più bravo, perché a lui bastano due versi per riassumere una narrazione epica. Che Marziale voglia presentarsi come un secondo Catullo anche nel modo particolare di trattare la materia epico-mitica, lo capiamo da un importante particolare: dal fatto, cioè, che l'epillio di Catullo (il c. 64) ha inizio proprio con

${ }^{20}$ In proposito cf. Galán Vioque (2002) 153. 
un'ampia descrizione della nave Argo e della sua difficile traversata (64.1-15):

Peliaco quondam prognatae vertice pinus dicuntur liquidas Neptuni nasse per undas Phasidos ad fluctus et fines Aeeteos, cum lecti iuvenes, Argivae robora pubis, auratam optantes Colchis avertere pellem ausi sunt vada salsa cita decurrere puppi, caerula verrentes abiegnis aequora palmis. Diva quibus retinens in summis urbibus arces ipsa levi fecit volitantem flamine currum, pinea coniungens inflexae texta carinae.

Illa rudem cursu prima imbuit Amphitriten; quae simul ac rostro ventosum proscidit aequor tortaque remigio spumis incanuit unda, emersere freti candenti e gurgite vultus aequoreae monstrum Nereides admirantes.

A Catullo, dunque, erano stati necessari 15 versi per illustrare un argomento che Marziale è stato capace di esporre in due soli versi! Sullo sfondo si delinea, al di là delle prese di posizione di poetica, anche una ironica giustificazione: è ovvio, infatti, che sia così, perché Catullo ha cantato la nave Argo nella sua integrità, Marziale, invece, solo un frammento di essa (v. 1 fragmentum)!

3.4. Ai più noti carmi dell'antichità appartiene senz'ombra di dubbio il c. 5 di Catullo:

Vivamus, mea Lesbia, atque amemus,

rumoresque senum severiorum

omnes unius aestimemus assis!

Soles occidere et redire possunt:

nobis cum semel occidit brevis lux,

nox est perpetua una dormienda.

Da mi basia mille, deinde centum,

dein mille altera, dein secunda centum,

deinde usque altera mille, deinde centum.

Dein, cum milia multa fecerimus,

conturbabimus illa, ne sciamus,

aut ne quis malus invidere possit,

cum tantum sciat esse basiorum. 
Il carme dei baci costituisce una proclamazione della forza dell'amore: nella descrizione che ne dà Catullo l'amore appare come l'unica barriera che l'uomo può frapporre, sia pure provvisoriamente, alla tragica brevità della propria esistenza, tanto diversa dalla vita della natura, dove il sole può morire ogni giorno per poi rinascere ogni giorno. Che al carme abbia arriso grande fortuna nell'antichità è Marziale stesso ad attestarcelo, sia in 6.34.7-8 nolo quot arguto dedit exorata Catullo / Lesbia: pauca cupit qui numerare potest sia in 12.59.1-3 tantum dat tibi Roma basiorum / post annos modo quindecim reverso / quantum Lesbia non dedit Catullo, entrambi imperniati sul motivo catulliano del dare basia, che Catullo aveva sviluppato nei vv. $7 \mathrm{sgg}$. In realtà l'epigramma 6.34 appartiene ai tre dedicati al motivo dei baci per il giovanetto Diadumeno, ${ }^{21}$ dove però Marziale preferisce ricollegarsi ai carmi catulliani per Giovenzio. ${ }^{22} \mathrm{Ma}$ che qui egli si serva anche del c. 5 è dimostrato dall'allusione diretta nei vv. 7-8 ai baci di Catullo per Lesbia e al motivo della somma dei baci che attira il malocchio (cf. Catullo 5.10-12). Esplicito, invece, è il richiamo al c. 5 di Catullo in 12.59.1-3.

Se questi due esempi ci attestano in modo chiaro la fortuna di cui godette il c. 5 di Catullo presso Marziale, le riprese allusive più interessanti nei confronti del carme catulliano dei baci sono negli epigrammi 5.20 e 11.6. Nel primo il poeta manifesta all'amico Giulio Marziale il proprio rincrescimento per la necessità di sottomettere la sua giornata ai doveri della clientela; non molto diversa, d'altronde, è la condizione dell'amico: intanto i giorni passano via veloci ed entrambi non potranno più recuperarli. In particolare si considerino i vv. 11-13:

nunc vivit necuter sibi bonosque

soles effugere atque abire sentit,

qui nobis pereunt et inputantur.

Se si pongono a confronto questi versi con quelli del modello, ci si rende conto della straordinaria raffinatezza dell'operazione compiuta da Marziale: egli ha individuato, non a caso, i versi più patetici del carme di Catullo, quelli in cui il poeta contrappone l'eterno ritmo della natura alla fugacità della vita umana, ma è stato capace di riutilizzarli conferendo loro un senso nuovo. In primo luogo, infatti, mentre i soles di Catullo

${ }^{21}$ Gli altri sono 3.65 e 5.46 .

${ }^{22}$ Si tratta dei carmi 48 e 99: cf. Grewing (1996) 333-356. 
rinviano alla luce sfolgorante del sole che ogni giorno rinasce (di qui il plurale soles) e s'oppongono all'oscurità della nox una perpetua, i boni soles di Marziale (con l'aggettivo accortamente separato dal sostantivo grazie all'enjambement) impongono che al sostantivo si accordi il suo significato metonimico di dies, che invece Catullo evita enfatizzando il motivo della luminosità. Soles occidere et redire possunt, proclama il v. 4 del carme catulliano: Marziale, dopo aver ripetuto soles sia pure in una diversa accezione, rimpiazza dapprima occidere con effugere, che da un lato è prosodicamente equivalente, dall'altro ha il vantaggio di sostituire alla fissità della morte (occidere) la dinamicità della fuga del tempo (effugere); successivamente muta il composto redire in un composto analogo ma antitetico in quanto al significato (abire), e lo fa per sottolineare il contrasto che esiste fra i ciclici ritorni del mondo naturale e il carattere unico e definitivo della vita dell'uomo. Per di più sentit, che sta al posto del catulliano possunt, esprime piena coscienza del divario fra l'esistenza dell'uomo e quella della natura. Nel v. 13, infine, mentre pereunt è il modo con cui Marziale allude a occidere e occidit di Catullo, il conclusivo inputantur (il verbo inputare appartiene al linguaggio finanziario e significa «mettere in conto») si ricollega al motivo della somma dei baci e alla necessità di non calcolarla con cui si chiude il carme catulliano: anche in questo caso, dunque, un rapporto allusivo implica un rovesciamento della posizione del modello.

Il secondo epigramma (11.6) trasferisce il lettore nella spensierata atmosfera della festa dei Saturnali; Marziale, dopo aver invitato uno schiavetto a versargli il vino, lo prega di dargli baci, ma 'catulliani': se, poi, i baci saranno numerosi quanto quelli di Catullo, gli farà dono del passer catulliano (vv. 14-16):

da nunc basia, sed Catulliana:

quae si tot fuerint quot ille dixit, donabo tibi passerem Catulli.

È chiaro che l'iniziale da nunc basia si ricollega a da mi basia che nel c. 5 di Catullo si trova ugualmente in inizio di verso (v. 7): per rendere inequivocabile che si tratta di un segnale allusivo, Marziale ha voluto specificare che desidera proprio baci alla catulliana maniera (baci speciali, dunque, perché innumerevoli). Che egli intenda rinviare proprio a questa caratteristica del contesto del modello lo si capisce dal v. 15, col chiaro ricordo del numero infinito di baci di cui parla Catullo. Dopo una 
tale premessa Marziale si concede la solita conclusione a sorpresa, di carattere chiaramente osceno: se il giovane schiavo farà quanto egli chiede, in cambio riceverà da Marziale il passer catulliano. Apparentemente si tratta di un dono letterario (i carmi 2-3 di Catullo sul passer di Lesbia); è evidente, però, che Marziale pensa a un dono di tutt'altra natura, giocando sull'ambivalenza semantica del termine passer che, com'è noto, nel linguaggio osceno diviene sinonimo di penis. ${ }^{23}$

3.5. Il c. 13 di Catullo è un biglietto d'invito a cena rivolto all'amico Fabullo: si tratta, però, di un invito a cena a dir poco singolare, perché Fabullo dovrà portare con sé tutto il necessario, mentre Catullo, a causa della sua povertà, sarà in grado di offrirgli solo la sua amicizia affettuosa e un profumo che, peraltro, appartiene a Lesbia:

Cenabis bene, mi Fabulle, apud me paucis, si tibi di favent, diebus, si tecum attuleris bonam atque magnam cenam, non sine candida puella et vino et sale et omnibus cachinnis.

Haec si, inquam, attuleris, venuste noster, cenabis bene; nam tui Catulli plenus sacculus est aranearum. Sed contra accipies meros amores seu quid suavius elegantiusve est: nam unguentum dabo, quod meae puellae donarunt Veneres Cupidinesque: quod tu com olfacies, deos rogabis totum ut te faciant, Fabulle, nasum.

Una serie di epigrammi di Marziale si ricollega in qualche modo al carme di Catullo, di cui riprende il motivo dell'invito povero per adattarlo anche a circostanze diverse dalla cena. Un normale invito a cena, ad esempio, è quello di 11.52, che si apre in modo identico al c. 13 di Catullo (Cenabis bene, mi Fabulle, apud me Cenabis belle, Iuli Cerialis, apud me); non stupisce che Marziale rinunci qui al motivo della sorpresa, perché l'invito è rivolto a Giulio Ceriale, a cui lo lega il rapporto del cliens

${ }^{23}$ Su Marziale 6.34 e Catullo cf. in particolare Schönberger (1995) 501-7; su Marziale 11.6 cf. il commento di Kay (1979) 43-44. 
nei confronti del patrono. Tutt'al più, quindi, Marziale potrà scusarsi per la modestia dei cibi e potrà sostenere che per avere da Giulio Ceriale una risposta positiva all'invito è disposto a inventarsi una serie di succulente portate; ma non potrà mai dire al suo patrono di portarsi la cena!

Dello stesso carattere del c. 13, invece, è l'epigramma 5.62: Marziale, nell'invitare un amico ai suoi giardini, lo esorta a portare anche tutto ciò che è necessario per un tranquillo riposo, perché non potrà contare né su divani da tempo sgangherati, né su inesistenti materassi, né su una rete valida. Marziale, insomma, ha comprato i giardini, ma chi vuole frequentarli deve pensare all'arredamento.

Iure tuo nostris maneas licet, hospes, in hortis, si potes in nudo ponere membra solo, aut si portatur tecum tibi magna supellex: nam mea iam digitum sustulit hospitibus.

Nulla tegit fractos - nec inanis - culcita lectos, putris et abrupta fascia reste iacet.

Sit tamen hospitium nobis commune duobus: emi hortos; plus est: instrue tu; minus est.

$\mathrm{Al}$ di là del motivo generale dell'invito povero, alcune analogie nei particolari rinviano in modo puntuale al modello.. ${ }^{24} \mathrm{Se}$, infatti, Catullo invita Fabullo a portare con sé bonam atque magnam cenam (vv. 3-4), l'amico di Marziale dovrà portare una magna supellex (v. 3); a si tecum attuleris di Catullo corrisponde, inoltre, si portatur tecum in Marziale. Entrambi, poi, si preoccupano di aggiungere la motivazione di un tanto singolare modo di agire: e, come Catullo la introduce nei vv. 7-8 con un nam esplicativo, così in Marziale la giustificazione dell'estrema povertà è introdotta da nam nel v. 4.

Il contenuto di 7.94 non è un invito a cena, ma uno scherzo su un profumo che ha la singolare proprietà di puzzare come la salsa di pesce se lo annusa il repellente Papilo:

Vnguentum fuerat, quod onyx modo parva gerebat: olfecit postquam Papylus, ecce, garumst.

Si può notare come Marziale riprenda nel brevissimo epigramma la conclusione del carme catulliano: infatti unguentum ...quod del v. 11 di

${ }^{24}$ Le elenca la Salanitro (1993-94) 288-9. 
Catullo ritorna nel v. 1 di Marziale e olfacies del v. 13 di Catullo in olfecit del v. 2 di Marziale.

Ma le allusioni possono celarsi anche in carmi che col contesto del modello non hanno nulla in comune: è questo il caso di uno degli Apophoreta (14.206): ${ }^{25}$ si tratta di un dono conviviale accompagnato da un bigliettino, che però è utile addirittura per risolvere un problema testuale presentato dal testo di Catullo:

Collo necte, puer, meros amores, ceston de Veneris sinu calentem.

È evidente che la clausola del v. 1 (meros amores) ripete quella del v. 9 del c. 13 di Catullo; gli apparati critici catulliani segnalano, a questo proposito, che c'è disaccordo nella tradizione manoscritta: se, infatti, il Parisinus Lat. 14137 (G) e il Vatic. Ottob. Lat. 1829 (R) tramandano meros, l'Oxoniensis Canonic. Class. Lat. $30(\mathrm{O})$ attesta invece meos. Che in Catullo la lezione giusta sia senz'ombra di dubbio quella di G R, di per sé preferibile perché difficilior nei confronti del banale meos, è confermato proprio dalla puntuale ripresa di Marziale.

Il caso più interessante, però, è quello di 3.12:

Vnguentum, fateor, bonum dedisti

convivis here, sed nihil scidisti.

Res salsa est bene olere et esurire.

Qui non cenat et unguitur, Fabulle, hic vere mihi mortuus videtur.

$\mathrm{Al}$ carme di Catullo rinvia subito il nome del destinatario (v. 4), che è lo stesso del c. 13 e costituisce un chiaro segnale dell'imitazione. Qui, però, la situazione è rovesciata, perché a invitare è stato Fabullo; il Fabullo di Marziale, però, si è comportato proprio come voleva comportarsi Catullo nel c. 13: ha dato ai suoi ospiti un unguentum ...bonum (cf. Catullo 13.11 per l'unguentum, mentre l'epiteto è trasferito dalla cena di Catullo al profumo); Fabullo, dunque, ha profumato ben bene i suoi ospiti, ma in compenso li ha lasciati morire di fame! Una ulteriore analogia risiede in res salsa est, che corrisponde all'uso metaforico dell'ablativo sale («facezie», «spiritosaggini») in Catullo (v. 5). ${ }^{26}$

25 Su Marziale 14.206 cf. Leary (1996) 274-5.

${ }^{26}$ Su Marziale 3.12 e il c. 13 di Catullo cf. Offermann (1980) 116-7. 
3.6. Nei 3 endecasillabi dell'epigramma 8.35 Marziale si rivolge a una uxor e a un maritus, entrambi pessimi - come nel v. 2 sottolinea il poliptoto in chiasmo - e osserva che, essendo a tal punto simili nel loro comportamento (v. 1), c'è proprio da stupirsi che non vadano d'accordo (v. 3):

Cum sitis similis paresque vita, uxor pessima, pessimus maritus, miror non bene convenire vobis.

In Catullo una coppia singolare è costituita da Giulio Cesare e dal suo favorito Mamurra, il bancarottiere di Formia. Nel c. 57 Catullo si prende gioco di entrambi servendosi di una serie di doppi sensi, che alludono a un loro stretto rapporto non solo politico-economico, ma anche erotico:

Pulcre convenit improbis cinaedis,

Mamurrae pathicoque Caesarique.

Nec mirum: maculae pares utrisque, urbana altera et illa Formiana, impressae resident nec eluentur:

morbosi pariter, gemelli utrique, uno in lectulo erudituli ambo, non hic quam ille magis vorax adulter, rivales socii puellularum.

Pulcre convenit improbis cinaedis.

Si vede bene come il verso iniziale del carme catulliano sia stato ripreso da quello finale dell'epigramma di Marziale: è bastato modificare pulcre in non bene e riproporre l'uso di convenire col dativo; per di più miror, che in Marziale apre il verso, deriva da nec mirum di 57.3. E ancora: l'insistenza di Marziale sulla totale identità nel modo di vivere dei due coniugi è la stessa di Catullo a proposito di Cesare e Mamurra (cf. v. 3 maculae pares utrisque, v. 6 morbosi pariter, gemelli utrique; e cf. anche i vv. 7-8). Quei lettori di Marziale che saranno stati capaci di stabilire il collegamento col carme catulliano ne avranno ricavato la giusta chiave di lettura: avranno capito, cioè, che quella coppia tanto ben assortita si era macchiata di ogni genere di nefandezze, soprattutto in campo sessuale.

3.7. Anche in altri casi è l'allusione a fornire la giusta chiave d'interpretazione. Consideriamo il ben noto c. 85 , in cui Catullo manifesta 
nei confronti di Lesbia un misto di disprezzo e di amore; egli se ne rende conto, ma non può farci nulla e se ne tormenta:

Odi et amo. Quare id faciam fortasse requiris?

Nescio, sed fieri sentio et excrucior.

Marziale tiene presente il c. 85 di Catullo in più d'una occasione, sia nella struttura sia nel contenuto, e sempre in carmi di un unico distico. È questo il caso di 5.83:

Insequeris, fugio; fugis, insequor. Haec mihi mens est: velle tuum nolo, Dindyme, nolle volo.

Il senso del carme è ben diverso, perché Marziale confessa di desiderare Dindimo quando costui gli resiste e di non desiderarlo quando è Dindimo a volerlo. Tuttavia è chiaro che l'epigramma di Marziale è costruito sull'identico sforzo di conciliazione degli opposti (insequeris, fugio; fugis, insequor; velle ...nolo; nolle volo) che caratterizza sin dall'esordio (odi et amo) il carme di Catullo. ${ }^{27}$

La stessa riflessione si può fare a proposito dell'epigramma 12.46, anch'esso interamente costruito sul tentativo di conciliare gli opposti:

Difficilis facilis, iucundus acerbus es idem: nec tecum possum vivere nec sine te.

Qui il tentativo è più complesso, perché il c. 85 di Catullo è presente nella struttura dell'esametro, mentre la fonte del pentametro è rappresentata da Ov. Am. 3.11 .39 sic ego nec sine te nec tecum vivere possum.

Il tentativo più complesso, però, è rappresentato da 1.32:

Non amo te, Sabidi, nec possum dicere quare:

hoc tantum possum dicere, non amo te.

Il legame col carme di Catullo non è solo nella struttura, ma anche nella ripresa di odi nel pressoché sinonimico non amo, che apre e chiude l'epigramma, e dell'interrogativa catulliana (quare id faciam fortasse requiris?) che è seguita poi dalla consapevolezza di non poter fornire una

27 Non se n'è accorto affatto, nel suo commento all'epigramma, Howell (1995) 163-4. 
spiegazione logica (nescio in Catullo, nec possum dicere quare in Marziale). Se all'allusione di Marziale si accorda il giusto peso, allora è probabile, come sostiene Citroni, che «Marziale voglia dire che egli non è in grado di esprimere il motivo del suo atteggiamento, perché è un atteggiamento istintivo».28

3.8. Si è già detto più sopra che un'allusione a Catullo 95.7-8 at Volusi Annales Paduam morientur ad ipsam / et laxas scombris saepe dabunt tunicas è in Marziale 3.2.3-5. Una ulteriore e ancor più evidente presenza del carme di Catullo è nell'epigramma 4.86, dove Marziale combina un'allusione a Catullo 95.7-8 con un'allusione a un altro carme di Catullo:

\author{
Si vis auribus Atticis probari \\ exhortor moneoque te, libelle, \\ ut docto placeas Apollinari: \\ nil exactius eruditiusque est, \\ sed nec candidius benigniusque. \\ Si te pectore, si tenebit ore, \\ nec rhonchos metuas maligniorum, \\ nec scombris tunicas dabis molestas: \\ si damnaverit, ad salariorum. \\ curras scrinia protinus licebit, \\ inversa pueris arande charta.
}

Nel rivolgersi al proprio libro di epigrammi, Marziale gli suggerisce d'incontrare l'approvazione del dotto Apollinare, giudice erudito e schietto. Se sarà di suo gradimento, allora non servirà per incartare gli sgombri; se invece, conclude Marziale, il dotto Apollinare emetterà un verdetto di condanna, il libro potrà finire nelle casse di un pescivendolo per essere adoperato dai ragazzi di bottega. Se è evidente la ripresa nel v. 8 di Catullo 95.8 et laxas scombris saepe dabunt tunicas, con la sola sostituzione di laxas con molestas, la sorpresa è nel finale, in cui Marziale rimane fedele a Catullo, ma non allo stesso carme di Catullo: dal c. 95, infatti, egli passa ora al c. 14.

Il c. 14 di Catullo è un tipico carme d'occasione, che costituisce la risposta del poeta a un dono singolare dell'amico e poeta Licinio Calvo in 125-6.

${ }^{28}$ Citroni (1975) 109; sul parallelo fra i due carmi cf. anche Offermann (1980) 
occasione della festa dei Saturnali; Calvo, per fare uno scherzo a Catullo, gli ha fatto dono di una raccolta di versi di pessimi poetae, e Catullo medita una giusta vendetta: non appena sorgerà il sole, si recherà nelle botteghe dei librarii e ricambierà il dono con un identico regalo dei versi più orrendi di poetastri contemporanei (14.17-20):

nam, si luxerit, ad librariorum

curram scrinia, Caesios, Aquinos,

Suffenum, omnia colligam venena

ac te his suppliciis remunerabor.

I vv. 17-18 di Catullo vengono ripresi pressoché integralmente nei vv. 910 di Marziale (si damnaverit, ad salariorum / curras scrinia): ma, come si può vedere, ciò avviene con una umoristica e degradante sostituzione dei librarii con i salarii (cioè con i pescivendoli, detti così perché salano il pesce per conservarlo) e delle casse (gli scrinia) che contenevano nobili e costosi papiri letterari con quelle, destinate ad accogliere ben altra cosa, delle botteghe dei venditori di pesce: in tal modo si fa ritorno al concetto già espresso nel c. 95 di Catullo (gli scritti di un pessimo poeta che servono da carta per avvolgere il pesce), ma vi si ritorna passando attraverso il c. 14 !

3.9. Il c. 101 di Catullo è un epigramma sepolcrale, che Catullo immagina di pronunciare sulla tomba del fratello nella Troade, al tempo del soggiorno in Bitinia al seguito di Memmio. Muovendo dallo schema dell'epigramma funebre, Catullo riesce a comporre un carme che va ben al di là delle convenzioni del genere ed esprime in modo profondo un sentimento intimo qual è il cordoglio per la morte del fratello:

Multas per gentes et multa per aequora vectus advenio has miseras, frater, ad inferias,

ut te postremo donarem munere mortis et mutam nequiquam alloquerer cinerem.

Quandoquidem fortuna mihi tete abstulit ipsum, heu miser indigne frater adempte mihi, nunc tamen interea haec, prisco quae more parentum. tradita sunt tristi munere ad inferias, accipe fraterno multum manantia fletu, atque in perpetuum, frater, ave atque vale. 
Un primo epigramma in cui Marziale mostra di ricollegarsi al carme catulliano è 1.88, che è un epitafio per Alcimo, un giovane schiavo del poeta rapito alla vita nel fiore degli anni:

Alcime, quem raptum domino crescentibus annis

Labicana levi caespite velat humus,

accipe non Pario nutantia pondera saxo, quae cineri vanus dat ruitura labor, sed faciles buxos et opacas palmitis umbras quaeque virent lacrimis roscida prata meis accipe, care puer, nostri monimenta doloris: hic tibi perpetuo tempore vivet honor.

Cum mihi supremos Lachesis perneverit annos, non aliter cineres mando iacere meos.

Non si può negare la presenza in Marziale di toni sinceri nell'espressione del sentimento di cordoglio per la morte del giovanetto: ma egli sapeva bene che in un epitafio doveva necessariamente misurarsi col più celebre esempio letterario del genere a Roma. Certo, non poteva fare inizialmente riferimento, come Catullo, alle peripezie di un lungo viaggio che l'aveva condotto sino alla tomba, ed era costretto a limitarsi al motivo delle offerte funebri. Catullo nei vv. 5-6 insiste nell'intendere la morte del fratello come una rapina compiuta ai suoi danni dal destino (v. 5 Fortuna mihi tete abstulit, v. 6 adempte mihi): Marziale riduce questa espressione del cordoglio a un semplice raptum domino, al quale, però, accorda peso grazie alla collocazione iniziale. A Catullo rimane fedele nel modo di presentare le offerte: accipe fraterno multum manantia fletu, aveva detto Catullo nel v. 9; nel v. 7 di Marziale le lacrime sono sostituite da un generico dolore, ma identico è il gesto (accipe, care puer, nostri monimenta doloris), che era già stato espresso nel v. 3 accipe non Pario nutantia pondera saxo. Parimenti significativo è che il v. 7 di Marziale sia seguito dalla formula del perpetuo ricordo (v. 8 hic tibi perpetuo tempore vivet honor), che si affianca anche lessicalmente a quella in cui Catullo prende congedo dal fratello augurandogli un eterno riposo (v. 10 atque in perpet u $\mathrm{m}$, frater, ave atque vale).

Tuttavia la suggestione del distico conclusivo del carme catulliano non si limita ad operare in 1.88: quando Marziale dovrà scrivere un carme di compianto per Camonio Rufo, morto durante un viaggio in Cappadocia poco prima della pubblicazione del VI libro degli 
epigrammi, ${ }^{29}$ egli si ricorderà del c. 101 di Catullo, anche per l'analogia della morte sia di Camonio Rufo sia del fratello di Catullo in terre lontane da Roma (6.85):

Editur en sextus sine te mihi, Rufe Camoni, nec te lectorem sperat, amici, liber:

impia Cappadocum tellus et numine laevo visa tibi cineres reddit et ossa patri.

Funde tuo lacrimas orbata Bononia Rufo, et resonet tota planctus in Aemilia:

heu qualis pietas, heu quam brevis occidit aetas!

Viderat Alphei praemia quinta modo.

Pectore tu memori nostros evolvere lusus, tu solitus totos, Rufe, tenere iocos,

accipe cum fletu maesti breve carmen amici atque haec absentis tura fuisse puta.

Marziale non può, come Catullo, fare visita alla tomba e deporre personalmente le offerte funebri: sarà allora la sua poesia a raggiungere l'amico che giace lontano e a sostituire l'incenso che si è soliti offrire ai defunti. E, perché sia chiaro che il suo gesto è lo stesso di Catullo nei confronti del fratello e come quello intensamente e profondamente affettivo, l'ultimo distico renderà inequivocabile il richiamo al modello: lo mostrano sia accipe in entrambi in inizio di verso, sia fraterno multum manantia fletu in Catullo e cum fletu maesti breve carmen amici in Marziale; e, perché non sussistano dubbi, dopoché l'ultimo esametro ha avuto inizio in entrambi con accipe, anche l'ultimo pentametro comincerà in entrambi allo stesso modo, con atque.

4. Ma è tempo di tirare le somme di questo discorso. Nel corso della sua attività di poeta epigrammatico Marziale capì bene che la sua denuncia della decadenza della società dava vita a una produzione letteraria di stampo realistico, la cui esigenza era vivamente avvertita da un ampio pubblico di lettori. Il successo che arrise ai suoi carmi lo convinse sempre più della necessità di trattare argomenti che trovavano una rispondenza reale nella vita di tutti i giorni. In origine i suoi carmi si ponevano un fine pratico occasionale, legati com'erano a momenti

${ }^{29}$ Sul personaggio cf. Grewing (1997) 543. 
particolari della vita dei suoi protettori o alla funzione d'intrattenimento nei conviti, nelle riunioni festose, nei momenti d'incontro della buona società. Ma l'ampliato pubblico dei lettori non comportava più l'esigenza esclusiva di una produzione artisticamente raffinata e rivolta solo a pochi intenditori: a questo nuovo pubblico, vasto, culturalmente non omogeneo e dagli interessi diversi, poteva legittimamente essere destinato un tipo di letteratura non caratterizzato da un'eccessiva difficoltà di scrittura, come nel caso della poesia epica o tragica. Nella difesa della poesia semplice e di brevi dimensioni, Marziale si rifà all'esperienza dei poeti del I sec. a.C. e non a caso cita esplicitamente Catullo quale modello dei suoi carmi. In questo egli si distacca volutamente dalle tendenze della sua epoca, nella quale l'erudizione e il poema di grandi dimensioni avevano avuto il sopravvento.

Contro l'epos Marziale sviluppa una polemica costante, rivendicando ai suoi versi il merito di saper giungere direttamente al cuore delle cose, senza troppi giri di parole (3.68), e di essere privi di ogni inutile ridondanza stilistica (4.69); i poemi epici, invece, sono da lui ritenuti bolse e futili esercitazioni su argomenti inconsistenti. Di essi egli rifiuta sia le tematiche mitologiche sia i contenuti ripetitivi, falsi e ormai privi d'incidenza nella realtà contemporanea, sia l'aspetto formale che li contraddistingue (lo stile gonfio e solenne). All'interno dei singoli carmi la struttura essenzialmente bipartita appare a tal punto chiara e lineare, che non ci si stupisce nel constatare che essa ha finito per influenzare in modo decisivo gli esiti moderni del genere epigrammatico: in una prima parte Marziale tende a creare una condizione di attesa nel lettore, presentandogli con tratti efficaci una situazione; in tal modo tutto è pronto per la conclusione a sorpresa, talora fulminea, in cui si concentra l'aspetto comico del carme.

Nell'esprimere la propria certezza in una sopravvivenza dei suoi epigrammi, Marziale manifestava una piena consapevolezza del valore letterario di una raccolta costituita senza precise connessioni fra i carmi all'interno dei singoli libri: ma proprio la continua variatio contenutistica era in grado di garantire il successo alle sue raccolte, che accontentavano le più diverse esigenze di quel nuovo pubblico di lettori, desiderosi di divertirsi ma anche di riflettere sulla realtà dei tempi, sui modi di comportamento, sui vizi più comuni della società. Di fronte alla sua produzione, però, i critici storcevano il naso, considerandola un prodotto minore di poesia, destinato a un piacevole intrattenimento o 
all'espressione di volgari denunce. A tali accuse Marziale cercò di rispondere, obiettando che i suoi carmi miravano a colpire i difetti, non le persone; ma non riuscì a placare le critiche dei letterati tradizionalisti, che consideravano con sospetto addirittura la struttura non sempre rigorosamente epigrammatica dei suoi carmi o l'adozione di schemi metrici diversi dal distico elegiaco. Ne è un esempio l'atteggiamento di Plinio il Giovane, che valuta la produzione epigrammatica di Marziale alla stessa stregua di un semplice divertimento momentaneo e ritiene del tutto spropositata la sua aspirazione all'immortalità. Nell'informare, infatti, l'amico Cornelio Prisco della morte di Marziale, Plinio cita i vv. 12-21 dell'epigramma 10.20 a lui rivolto e commenta (Epistole 3.21): dedit enim mihi quantum maximum potuit, daturus amplius si potuisset. Tametsi quid homini potest dari maius, quam gloria et laus et aeternitas? At non erunt aeterna quae scripsit: non erunt fortasse, ille tamen scripsit tamquam essent futura («egli mi ha dato ciò che poteva darmi di meglio; se avesse potuto, mi avrebbe dato di più. D'altronde, che cosa si può dare a un uomo di più della gloria, della lode e dell'immortalità? Non saranno eterni i versi che scrisse? Forse non lo saranno, ma egli li ha scritti come se dovessero esserlo").

Ecco che allora la preferenza accordata da Marziale ai carmi di Catullo acquista un senso ben preciso, perché si configura come una consapevole e orgogliosa rivendicazione di validità per un genere di poesia solo apparentemente minore: Marziale lo afferma esplicitamente quando proclama (10.78.16) di essere secondo solo a Catullo nella lista degli esponenti del genere epigrammatico ed esprime la certezza di aver raggiunto quell'immortalità che è concessa solo ai poeti grandi. Il suo è un aperto atto di sfida nei confronti dei letterati alla moda, che continuano a considerare l'epigramma come un genere inferiore.

Da Catullo, comunque, egli sa anche prendere le distanze, perché rispetto all'esperienza catulliana, gli epigrammi di Marziale rappresentano la società che si muove intorno al poeta, più che la sua interiorità: a tal fine acquista grande importanza la battuta finale, nella quale il poeta condensa l'effetto comico e la ricerca di esiti brillanti ed inaspettati. Ciò avviene anche in carmi che presentano un contenuto serio, a cui corrisponde un tono talora solenne che viene poi sovvertito nel verso conclusivo. Il carme 5.37 è un epicedio che celebra, nel metro scazonte e con accenti di patetico e doloroso rimpianto, la morte di Erotion, la schiavetta tanto cara al poeta, che non aveva ancora compiuto sette anni; 
in esso confluiscono la sensibilità per una morte prematura e sentimenti di cordialità e di affetto nei confronti di una vita troppo presto troncata:

Puella senibus dulcior mihi cycnis,
agna Galaesi mollior Phalantini,
concha Lucrini delicatior stagni,
cui nec lapillos praeferas Erythraeos
nec modo politum pecudis Indicae dentem
nivesque primas liliumque non tactum;
quae crine vicit Baetici gregis vellus
Rhenique nodos aureamque nitelam;
fragravit ore quod rosarium Paesti,
quod Atticarum prima mella cerarum,
quod sucinorum rapta de manu gleba;
cui comparatus indecens erat pavo,
inamabilis sciurus et frequens phoenix:
adhuc recenti tepet Erotion busto,
quam pessimorum lex amara fatorum
sexta peregit hieme, nec tamen tota,
nostros amores gaudiumque lususque;
et esse tristem me meus vetat Paetus,
pectusque pulsans pariter et comam vellens:
"Deflere non te vernulae pudet mortem?
ego coniugem" inquit "extuli et tamen vivo,
notam, superbam, nobilem, locupletem".
Quid esse nostro fortius potest Paeto?
ducenties accepit et tamen vivit.

Le doti straordinarie di Erotion sono qui esaltate attraverso una lunga serie di paralleli: agli effetti a sorpresa dell'epigramma appartiene la scoperta, che avviene solo al v. 16, che quella che sembrava una puella è in realtà una bimba da poco defunta, di cui Marziale nei vv. 14-17 ricapitola i tratti fondamentali, conferendo ai quattro versi la struttura di un breve epicedio (vv. 14-16 la morte prematura; v. 17 l'affetto del poeta per la defunta). Ora, finalmente, il lettore riesce a capire il motivo della presenza dei perfetti nei vv. 7 e 9 e dell'imperfetto nel v. 12 e di alcuni singolari paralleli (il cigno morente, il giglio, la fenice). Non stupisce nel carme la presenza di espressioni della sfera erotica, perché il linguaggio latino degli affetti ha un'ampia sfera di applicazione, e si adatta sia all'amore, sia all'amicizia, sia ai legami familiari. La società romana, però, 
riteneva sconveniente un'eccessiva manifestazione di cordoglio per la morte di uno schiavo: ecco perché la morte di Erotion diviene l'occasione per un attacco contro un rivale, Peto, che simile a un predicatore stoico cerca di consolare Marziale ricordandogli il suo lutto ben più grave per la morte della moglie. L'introduzione del nuovo personaggio al v. 18 provoca un ironico innalzamento dello stile (v. 18 me meus; v. 19 la solenne allitterazione trimembre pectusque pulsans pariter; vv. 20-21, nell'invito a vergognarsi delle manifestazioni di lutto, l'intensivo deflere e le giustapposizioni dello stile affettivo te vernulae ed ego coniugem; v. 21 et tamen collocato fra i due estremi antitetici rappresentati da extuli e vivo). Il lettore, però, si rende subito conto del diverso modo di procedere nell'elogio della persona defunta, perché alla lunga lista di elogi di Erotion fa riscontro una totale assenza di termini affettivi nella presentazione della moglie di Peto, di cui il marito liquida le doti con una rapida successione asindetica nel v. 22, che suona ironicamente solenne. Da bravo tradizionalista, dunque, Peto oppone al dolore di Marziale per la morte di Erotion la sua virile sopportazione del grave lutto familiare. Bella scoperta, sbotta spazientito Marziale! In realtà Peto è un volgare cacciatore di testamenti, perché per lui la morte della moglie ha comportato un notevole incremento delle sostanze (v. 24 ducenties accepit et tamen vivit)!

5. Un imitatore, quindi? Certo, e non del solo Catullo. Tuttavia nel caso suo, come sempre nella letteratura dell'antichità, imitare non significa semplicemente riprodurre: i modelli esistono, per Marziale come per ogni autore latino, e di essi non può fare a meno una cultura che su di essi si fonda: ma ricrearli e competere con essi in forma più o meno apertamente allusiva rappresenta il massimo omaggio che l'imitatore può offrire ai suoi modelli. 


\section{Bibliografia}

BonvicinI, Mariella: "Note sui «comparativi» in clausola nel falecio di Marziale", BStudLat 16 (1986) 31-35.

Bonvicini, Mariella: "Il faselo catulliano da Properzio a Marziale", BStudLat 25 (1995) 3-15.

Citroni, Mario: M. Valerii Martialis Epigrammaton Liber I (Firenze, 1975).

Ferguson, J.: "Catullus and Martial", PAfrClassAss 6 (1963) 3-15.

Galán Vioque, Guillermo: Martial. Book VII. A Commentary (Leiden-Boston-Köln, 2002).

GrewING, Farouk: "Möglichkeiten und Grenzen des Vergleichs: Martials 'Diadumenos' und Catulls 'Lesbia'", Hermes 124 (1996) 333-356.

GREWING, Farouk: Martial. Buch VI. Ein Kommentar (Göttingen, 1997).

HAllETT, Judith P.: “'Nec castrare velis meos libellos'. Sexual and Poetic 'lusus' in Catullus, Martial and the 'Carmina Priapea'", in Satura lanx. Festschrift für W.A. Krenkel (Hildesheim, 1996), 321-344.

HENRIKSÉN, Christer: Martial. Book IX. A Commentary (Uppsala, 1998-9).

Howell, Peter: Martial. The Epigrams. Book V (Warminster, 1995).

KAY, N.M.: A Commentary on Martial XI (Oxford, 1979).

LEARY, T.J.: Martial. Book XIV. The Apophoreta (London, 1996).

NEWMaN, John Kevin: Roman Catullus and the Modification of the Alexandrian Sensibility (Hildesheim, 1990).

OFFERMANN, Helmut: "'Uno tibi sim minor Catullo'", QUCC 5 (1980) 107-139.

PAUKSTADT, Rudolf: De Martiale Catulli imitatore (Halle, 1876).

SAlAnITRO, Maria: "Le tappe di un motivo folclorico (da Catullo ai giorni nostri)", InvLuc 15-16 (1993-94) 285-9.

SALEMME, Carmelo: Marziale e la poetica degli oggetti (Napoli, 1976).

SCHÖnBerger, Otto: "Oppositio in imitando. Zu Martial 6,34", Gymnasium 102 (1995) 501-7.

SWANN, Bruce W.: "'Sic scribit Catullus': The Importance of Catullus for Martial's Epigrams", in Toto notus in orbe. Perspektiven der Martial-Interpretation, ed. F. Grewing (Stuttgart, 1998), 48-58. 